NBER WORKING PAPER SERIES

\title{
STRATEGIC ASSET ALLOCATION IN A CONTINUOUS-TIME VAR MODEL
}

\author{
John Y. Campbell \\ George Chacko \\ Jorge Rodriguez \\ Luis M. Viciera \\ Working Paper 9547 \\ http://www.nber.org/papers/w9547 \\ NATIONAL BUREAU OF ECONOMIC RESEARCH \\ 1050 Massachusetts Avenue \\ Cambridge, MA 02138 \\ March 2003
}

Campbell acknowledges the financial support of the National Science Foundation, and Chacko and Viceira acknowledge the financial support of the Division of Research of the Harvard Business School. We are grateful to David Bates for challenging us to explore the consistency of continuous-time and discrete-time solution methods for VAR portfolio choice problems. The views expressed herein are those of the authors and not necessarily those of the National Bureau of Economic Research.

(C2003 by John Y. Campbell, George Chacko, Jorge Rodriguez, Luis M. Viciera. All rights reserved. Short sections of text not to exceed two paragraphs, may be quoted without explicit permission provided that full credit including Cnotice, is given to the source. 
Strategic Asset Allocation in a Continuous-Time VAR Model

John Y. Campbell, George Chacko, Jorge Rodriguez, Luis M. Viciera

NBER Working Paper No. 9547

March 2003

JEL No. G12

\section{$\underline{\text { ABSTRACT }}$}

This note derives an approximate solution to a continuous-time intertemporal portfolio and consumption choice problem. The problem is the continuous-time equivalent of the discrete-time problem studied by Campbell and Viceira (1999), in which the expected excess return on a risky asset follows an $\mathrm{AR}(1)$ process, while the riskless interest rate is constant. The note also shows how to obtain continuous-time parameters that are consistent with discrete-time econometric estimates. The continuous-time solution is numerically close to that of Campbell and Viceira and has the property that conservative long-term investors have a large positive intertemporal hedging demand for stocks.

John Y. Campbell

Department of Economics

Littauer Center 213

Harvard University

Cambridge, MA 02138

and NBER

john_campbell@harvard.edu

Jorge Rodriguez

Sloan School of Management

E52-458

50 Memorial Drive

Cambridge, MA 02142

jorge.rodriguez@sloan.mit.edu
George Chacko

Harvard Business School

Morgan Hall 389

Boston, MA 02163

gchacko@hbs.edu

Luis M. Viciera

Harvard Business School

Morgan Hall 395

Boston, MA 02163

and NBER

lviciera@hbs.edu 


\section{Introduction}

Campbell and Viceira (1999) study the impact of predictable variation in stock returns on intertemporal optimal portfolio choice and consumption. They consider an infinitely lived investor who faces a constant riskless interest rate and a time-varying equity premium. They model this time-variation using a discrete-time, homoskedastic VAR (1) process for log excess stock returns and a state variable driving changes in expected returns. This model of investment opportunities implies that the Sharpe ratio is linear in the state variable.

Campbell and Viceira assume that the investor has recursive Epstein-Zin utility (Epstein and Zin 1989, 1991), a generalization of power utility that allows both the coefficient of relative risk aversion and the elasticity of intertemporal substitution in consumption to be constant free parameters. They derive an approximate analytical solution for the optimal portfolio rule, and show that this rule is linear in the state variable. When they calibrate this model to U.S. stock market data for the postwar period, they find that intertemporal hedging motives greatly increase the average demand for stocks by investors whose relative risk aversion coefficients exceed one.

Because Campbell and Viceira work in discrete time, no exact portfolio solutions are available in their model except in the trivial case of unit risk aversion, which implies myopic portfolio choice. Campbell and Viceira claim, however, that their solution becomes exact in the limit of continuous time when the elasticity of intertemporal substitution equals one. They base this claim on the fact that they use an approximation to the investor's intertemporal budget constraint which becomes exact as the time interval of their model shrinks.

This paper presents a continuous-time analysis of Campbell and Viceira's portfolio choice problem. The paper finds a continuous-time representation of the VAR(1) process in their paper, and solves a continuous-time version of their model. The solution is exact when 
the elasticity of intertemporal substitution equals one, and approximate otherwise. The continuous-time solution has the same qualitative properties as the discrete-time solution in Campbell and Viceira, and is quantitatively similar. However the continuous-time solution is likely to be more appealing and intuitive to finance theorists who are accustomed to working in continuous time.

\section{Investment Opportunity Set}

\subsection{A continuous-time VAR}

We start by assuming that there are two assets available to the investor, a riskless asset with instantaneous return

$$
\frac{d B_{t}}{B_{t}}=r d t
$$

and a risky asset ("stocks") whose instantaneous return and expected return follow a continuoustime bivariate process:

$$
\begin{aligned}
{\left[\begin{array}{c}
d\left(\log S_{t}+\frac{1}{2} \sigma_{S}^{2} t-\theta t\right) \\
d\left(\mu_{t}-\theta\right)
\end{array}\right]=} & {\left[\begin{array}{cc}
0 & 1 \\
0 & -\kappa
\end{array}\right]\left[\begin{array}{c}
\log S_{t}+\frac{1}{2} \sigma_{S}^{2} t-\theta t \\
\mu_{t}-\theta
\end{array}\right] d t } \\
& +\left[\begin{array}{cc}
\sigma_{S} & 0 \\
\rho \sigma_{\mu} & \sqrt{\sigma_{\mu}^{2}-\rho^{2} \sigma_{\mu}^{2}}
\end{array}\right]\left[\begin{array}{c}
d Z_{S, t} \\
d Z_{\mu, t}
\end{array}\right],
\end{aligned}
$$

where $d Z_{S, t}$ and $d Z_{\mu, t}$ are independent Wiener processes.

Equation (2) implies that the instantaneous return on stocks $\left(d S_{t} / S_{t}\right)$ follows a Geometric Brownian Motion, whose drift (or instantaneous expected return) $\mu_{t}$ follows a mean-reverting process. Section 2.3 below shows that this is the continuous-time counterpart of the discretetime VAR(1) process in Campbell and Viceira (1999). 
We can write (2) in compact form as

$$
d y_{t}=A y_{t} d t+C d Z_{t}
$$

Note that the instantaneous variance of $d y$ is given by $C C^{\prime}$ :

$$
\operatorname{Var}(d y)=C C^{\prime}=\left[\begin{array}{cc}
\sigma_{S}^{2} & \rho \sigma_{S} \sigma_{\mu} \\
\rho \sigma_{S} \sigma_{\mu} & \sigma_{\mu}^{2}
\end{array}\right]
$$

\subsection{Time-aggregation of the continuous-time VAR}

Bergstrom (1984) and Campbell and Kyle (1993) show how to derive the discrete-time process implied by a continuous-time VAR when we take point observations of the continuous time process at evenly spaced points $\left\{t_{0}, t_{1} \ldots, t_{n}, t_{n+1}, \ldots\right\}$, with $\Delta t=t_{n}-t_{n-1}$. Direct application of their results shows that the process $y$ in (3) has the following discrete-time $\operatorname{VAR}(1)$ representation:

$$
y_{t_{n}+\Delta t}^{p}=\exp \{\Delta t A\} y_{t_{n}}^{p}+u_{t_{n}+1}^{p},
$$

where

$$
u_{t_{n}+1}^{p}=\int_{\tau=0}^{\Delta t} \exp \{(\Delta t-\tau) A\} C d Z_{t_{n}+\tau},
$$

and

$$
\exp \{A\}=I+\sum_{r=1}^{\infty} \frac{A^{r}}{r !}
$$

We prove in Appendix A that $\exp \{s A\}$ is equal to

$$
\exp (A s)=\left[\begin{array}{cc}
1 & \frac{1}{\kappa}\left(1-e^{-\kappa s}\right) \\
0 & e^{-\kappa s}
\end{array}\right]
$$

Thus we can write (4) in matrix form as:

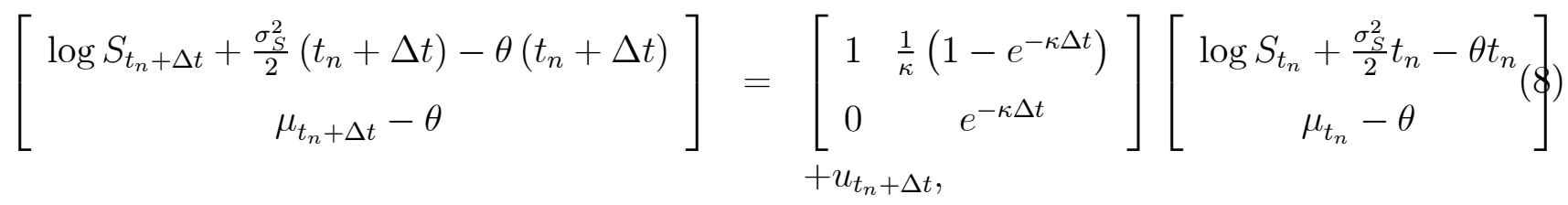


where

$$
u_{t_{n}+\Delta t}=\left[\begin{array}{c}
u_{S, t_{n}+\Delta t} \\
u_{\mu, t_{n}+\Delta t}
\end{array}\right]=\int_{0}^{\Delta t}\left[\begin{array}{cc}
1 & \frac{1}{\kappa}\left(1-e^{-\kappa(\Delta t-\tau)}\right) \\
0 & e^{-\kappa(\Delta t-\tau)}
\end{array}\right]\left[\begin{array}{cc}
\sigma_{S} & 0 \\
\rho \sigma_{\mu} & \sqrt{\sigma_{\mu}^{2}-\rho^{2} \sigma_{\mu}^{2}}
\end{array}\right] d Z_{t_{n}+\tau} .
$$

$>$ From equation (9), it follows that the variance-covariance matrix of the innovations $u_{t_{n}+\Delta t}$ in the discrete-time representation of the continuous-time VAR is given by

$$
\begin{aligned}
\operatorname{Var}\left(u_{t_{n}+\Delta t}^{p}\right) & =\int_{\tau=0}^{\Delta t} \exp \{(\Delta t-\tau) A\} C C^{\prime} \exp \left\{(\Delta t-\tau) A^{\prime}\right\} d \tau \\
& =\int_{0}^{\Delta t}\left[\begin{array}{cc}
B_{11} & B_{12} \\
B_{12} & B_{22}
\end{array}\right] d \tau
\end{aligned}
$$

where

$$
\begin{aligned}
& B_{11}=\sigma_{S}^{2}+\frac{2 \rho \sigma_{S} \sigma_{\mu}}{\kappa}\left(1-e^{-\kappa(\Delta t-\tau)}\right)+\frac{\sigma_{\mu}^{2}}{\kappa^{2}}\left(1-e^{-\kappa(\Delta t-\tau)}\right)^{2}, \\
& B_{12}=\rho \sigma_{S} \sigma_{\mu} e^{-\kappa(\Delta t-\tau)}+\frac{\sigma_{\mu}^{2}}{\kappa}\left(e^{-\kappa(\Delta t-\tau)}-e^{-2 \kappa(\Delta t-\tau)}\right), \\
& B_{22}=\sigma_{\mu}^{2} e^{-2 \kappa(\Delta t-\tau)} .
\end{aligned}
$$

Therefore, given values for the parameters of the continuous-time process (2), we can easily aggregate to any frequency $\Delta t$, by using (8) and (10). The discrete-time representation is especially useful in recovering the parameters of the continuous-time VAR (2) from estimates of the equivalent discrete-time VAR (8). We do this in the next section.

\subsection{Recovering continuous-time parameters from a discrete-time VAR}

In their analysis of optimal consumption and portfolio choice with time-varying expected returns, Campbell and Viceira $(1999,2000)$ assume that the log excess returns on stocks is 
described by the following discrete-time $\operatorname{VAR}(1)$ :

$$
\left[\begin{array}{c}
\Delta \log S_{t_{n}+\Delta t}-r_{f} \\
x_{t_{n}+\Delta t}
\end{array}\right]=\left[\begin{array}{c}
0 \\
(1-\phi) \mu
\end{array}\right]+\left[\begin{array}{ll}
0 & 1 \\
0 & \phi
\end{array}\right]\left[\begin{array}{c}
\Delta \log S_{t_{n}}-r_{f} \\
x_{t_{n}}
\end{array}\right]+\left[\begin{array}{c}
\varepsilon_{t_{n}+\Delta t} \\
\eta_{t_{n}+\Delta t}
\end{array}\right],
$$

where $r_{f}$ is the return (assumed constant) on a T-bill with maturity $\Delta t$.

We now show that the discrete-time VAR given in (11) and the continuous-time VAR given in (2) are equivalent representations of the same process. To see this, note that we can rewrite the discrete-time aggregation of $y$ in (8) as follows:

$$
\begin{aligned}
{\left[\begin{array}{c}
\Delta \log S_{t_{n}+\Delta t}-r \Delta t \\
\mu_{t_{n}+\Delta t}
\end{array}\right]=} & {\left[\begin{array}{c}
\left(\theta-\frac{\sigma_{S}^{2}}{2}-r\right) \Delta t-\frac{1}{\kappa}\left(1-e^{-\kappa \Delta t}\right) \theta \\
\left(1-e^{-\kappa \Delta t}\right) \theta
\end{array}\right] } \\
+ & {\left[\begin{array}{cc}
1 & \frac{1}{\kappa}\left(1-e^{-\kappa \Delta t}\right) \\
0 & e^{-\kappa \Delta t}
\end{array}\right]\left[\begin{array}{c}
\log S_{t_{n}}+\frac{\sigma_{S}^{2}}{2} t_{n}-\theta t_{n} \\
\mu_{t_{n}}
\end{array}\right]+\left[\begin{array}{l}
u_{S, t_{n}+\Delta t} \\
u_{\mu, t_{n}+\Delta t}
\end{array}\right] . }
\end{aligned}
$$

Using the following linear transformation for the process $\mu_{t}$,

$$
v_{t}=\left(\theta-\frac{\sigma_{S}^{2}}{2}-r\right) \Delta t-\frac{1}{\kappa}\left(1-e^{-\kappa \Delta t}\right) \theta+\frac{1}{\kappa}\left(1-e^{-\kappa \Delta t}\right) \mu_{t}
$$

we can further rewrite (12) in the same form as (11):

$$
\begin{aligned}
{\left[\begin{array}{c}
\Delta \log S_{t_{n}+\Delta t}-r \Delta t \\
v_{t_{n}+\Delta t}
\end{array}\right]=} & {\left[\begin{array}{c}
0 \\
\left(1-e^{-\kappa \Delta t}\right)\left(\theta-\frac{\sigma_{S}^{2}}{2}-r\right) \Delta t
\end{array}\right] } \\
& +\left[\begin{array}{cc}
0 & 1 \\
0 & e^{-\kappa \Delta t}
\end{array}\right]\left[\begin{array}{c}
\Delta \log S_{t_{n}+\Delta t}-r \Delta t \\
v_{t_{n}}
\end{array}\right] \\
& +\left[\begin{array}{cc}
1 & 0 \\
0 & \frac{1}{\kappa}\left(1-e^{-\kappa \Delta t}\right)
\end{array}\right]\left[\begin{array}{c}
u_{S, t_{n}+\Delta t} \\
u_{\mu, t_{n}+\Delta t}
\end{array}\right] .
\end{aligned}
$$

A simple comparison of the coefficients in (11) and (13) gives us a system of equations that relate the discrete-time parameters of the VAR process in Campbell and Viceira (1999) to the continuous-time parameters of our continuous time VAR process. For the intercept 
and slope parameters we have the following equivalence relations:

$$
\begin{aligned}
r_{f} & =r \Delta t, \\
\mu & =\left(\theta-\frac{\sigma_{S}^{2}}{2}-r\right) \Delta t, \\
\phi & =e^{-\kappa \Delta t} .
\end{aligned}
$$

Finally, using (10), we obtain the following equivalence relations for the variance and covariance parameters:

$$
\begin{aligned}
\operatorname{Var}_{t_{n}}\left(\eta_{t_{n}+\Delta t}\right)= & \frac{1}{\kappa^{2}}\left(1-e^{-\kappa \Delta t}\right)^{2} \operatorname{Var}_{t_{n}}\left(u_{\mu, t_{n}+\Delta t}\right) \\
= & \frac{\sigma_{\mu}^{2}}{2 \kappa^{3}}\left(1-e^{-\kappa \Delta t}\right)^{2}\left(1-e^{-2 \kappa \Delta t}\right), \\
\operatorname{Cov}_{t_{n}}\left(\varepsilon_{t_{n}+\Delta t}, \eta_{t_{n}+\Delta t}\right)= & \frac{1}{\kappa}\left(1-e^{-\kappa \Delta t}\right) \operatorname{Cov}_{t}\left(u_{S, t_{n}+\Delta t}, u_{\mu, t_{n}+\Delta t}\right) \\
= & \frac{\rho \sigma_{S} \sigma_{\mu}}{\kappa^{2}}\left(1-e^{-\kappa \Delta t}\right)^{2}+\frac{\sigma_{\mu}^{2}}{\kappa^{3}}\left(1-e^{-\kappa \Delta t}\right)^{2} \\
& -\frac{\sigma_{\mu}^{2}}{2 \kappa^{3}}\left(1-e^{-2 \kappa \Delta t}\right)\left(1-e^{-\kappa \Delta t}\right), \\
= & \operatorname{Var}_{t_{n}}\left(u_{S, t_{n}+\Delta t}\right) \\
\operatorname{Var}_{t_{n}}\left(\varepsilon_{t_{n}+\Delta t}\right) & \left(\sigma_{S}^{2}+\frac{2 \rho \sigma_{S} \sigma_{\mu}}{\kappa}+\frac{\sigma_{\mu}^{2}}{\kappa^{2}}\right) \Delta t-\frac{2 \rho \sigma_{S} \sigma_{\mu}}{\kappa^{2}}\left(1-e^{-\kappa \Delta t}\right) \\
& -\frac{2 \sigma_{\mu}^{2}}{\kappa^{3}}\left(1-e^{-\kappa \Delta t}\right)+\frac{\sigma_{\mu}^{2}}{2 \kappa^{3}}\left(1-e^{-2 \kappa \Delta t}\right) .
\end{aligned}
$$

Campbell-Viceira (2000) report estimates of the VAR (11) based on US quarterly data for the period 1947.Q1-1995.Q4. Table I shows the value of the parameters of the continuoustime equivalent VAR implied by their estimates. ${ }^{2}$

\footnotetext{
${ }^{2}$ There is an estimation error in Campbell and Viceira (1999) that results in an underestimation of the degree of predictability in stock returns in their paper. Campbell and Viceira (2000) report correct estimates, and calibration results based on the corrected estimates.
} 


\subsection{A common mistake}

Anyone used to working with the discrete-time representation of a univariate continuoustime process will find natural and intuitive the relation between the intercept and slope of the continuous-time VAR and its discrete-time representation implied by equations (14)-(16). However, equations (17)-(19) show that the equivalence relation for the variance-covariance matrix of innovations is less obvious. Using an intuitive extension of the usual matching rules for simple, univariate process, one might be tempted to write:

$$
\begin{aligned}
\operatorname{Var}_{t_{n}}\left(\varepsilon_{n+1}\right) & \approx \sigma_{S}^{2} \Delta t \\
\operatorname{Cov}_{t_{n}}\left(\varepsilon_{t_{n}+\Delta t}, \eta_{t_{n}+\Delta t}\right) & \approx \rho \sigma_{S} \sigma_{\mu} \Delta t \\
\operatorname{Var}_{t_{n}}\left(\eta_{t_{n}+\Delta t}\right) & \approx \sigma_{\mu}^{2} \Delta t .
\end{aligned}
$$

It should be apparent from equations (17)-(19) that this matching is incorrect- - though equation (20) is a first-order Taylor expansion of the correct expression for $\operatorname{Var}_{t_{n}}\left(\varepsilon_{n+1}\right)$ given in (19). The use of (20) is particularly dangerous when $\Delta t \neq 1$, as might be the case when one is using annualized parameters and quarterly data. In this case portfolio solutions based on (20) can be quite different from the correct solutions that we will derive using equations (17)-(19).

\section{Intertemporal Portfolio Choice}

We have shown in Section 2, that the investment opportunity set described by equations (1) and (2) is equivalent to the investment opportunity set that Campbell and Viceira (1999) assume in their discrete-time, intertemporal optimal consumption and portfolio choice model. In this section we solve their model in continuous time, using the techniques described in Chacko and Viceira (2000) and Campbell and Viceira (2002), and we show that the 
solution is invariant to the choice of discrete-time or continuous-time approximations to solve for the model.

\subsection{Assumptions on investment opportunities and preferences}

We consider an investor who has only two assets available for investment, a riskless bond and stocks, and no labor income. Return dynamics are given by (1) and the bivariate system (2). For convenience, we rewrite the system (2) as

$$
\frac{d S_{t}}{S_{t}}=\mu_{t} d t+\sigma_{S} d \widetilde{Z}_{S}
$$

where

$$
d \mu_{t}=\kappa\left(\theta-\mu_{t}\right) d t+\sigma_{\mu} d \widetilde{Z}_{\mu}
$$

where $d \widetilde{Z}_{S}=d Z_{S}$, and $d \widetilde{Z}_{\mu}=\rho d Z_{S}+\sqrt{1-\rho^{2}} d Z_{\mu}$. Note that the instantaneous correlation between $d S_{t} / S_{t}$ and $d \mu_{t}$ is $\rho$.

These assumptions on investment opportunities imply that the wealth dynamics for the investor are given by

$$
d W_{t}=r W_{t} d t+\alpha_{t} W_{t}\left[\left(\mu_{t}-r\right) d t+\sigma_{S} d Z_{S}\right]-C_{t} d t
$$

where $\alpha_{t}$ is the fraction of wealth invested in stocks.

Campbell and Viceira (1999) assume that the investor has recursive Epstein-Zin preferences over consumption. Duffie and Epstein (1992a, b) provide an equivalent continuous-time parameterization of recursive preferences:

$$
J_{t}=\int_{t}^{\infty} f\left(C_{s}, J_{s}\right) d s
$$

where $f\left(C_{s}, J_{s}\right)$ is a normalized aggregator of current consumption and continuation utility 
that takes the form

$$
f(C, J)=\frac{\beta}{1-\frac{1}{\psi}}(1-\gamma) J\left[\left(\frac{C}{((1-\gamma) J)^{\frac{1}{1-\gamma}}}\right)^{\left(1-\frac{1}{\psi}\right)}-1\right] .
$$

Here $\beta>0$ is the rate of time preference, $\gamma>0$ is the coefficient of relative risk aversion, and $\psi>0$ is the elasticity of intertemporal substitution.

There are two interesting special cases of the normalized aggregator (22): $\psi=1 / \gamma$ and $\psi=1$. The case $\psi=1 / \gamma$ is interesting because in that case the normalized aggregator $(22)$ reduces to the standard, additive power utility function - from which log utility obtains by setting $\gamma=1$. In the second special case, the aggregator $f\left(C_{s}, J_{s}\right)$ takes the following form as $\psi \rightarrow 1$ :

$$
f\left(C_{s}, J_{s}\right)=\beta(1-\gamma) J\left[\log (C)-\frac{1}{1-\gamma} \log ((1-\gamma) J)\right] .
$$

The case $\psi=1$ is important because it allows an exact solution to our dynamic optimization

problem for investors who are more risk averse than an investor with unit coefficient of relative risk aversion. We now explore this solution, as well as an approximate solution for investors with $\psi \neq 1$ in the next section.

\subsection{Bellman equation}

Duffie and Epstein (1992a, b) show that the standard Bellman principle of optimality applies to recursive utility. The Bellman equation for this problem is

$$
\begin{aligned}
0= & \sup _{\left\{\alpha_{t}, C_{t}\right\}}\left\{f\left(C_{t}, J_{t}\right)+J_{W}\left[W_{t}\left(r+\alpha_{t}\left(\mu_{t}-r\right)\right)-C_{t}\right]+J_{\mu} \kappa\left(\theta-\mu_{t}\right)\right. \\
& \left.+\frac{1}{2} J_{W W} W_{t}^{2} \alpha_{t}^{2} \sigma_{S}^{2}+J_{W \mu} W_{t} \alpha_{t} \rho \sigma_{S} \sigma_{\mu}+\frac{1}{2} J_{\mu \mu} \sigma_{\mu}^{2}\right\}
\end{aligned}
$$

where $f\left(C_{t}, J_{t}\right)$ is given in $(22)$ when $\psi \neq 1$, or $(23)$ when $\psi=1 . J_{x}$ denotes the partial derivative of $J$ with respect to $x$, except $J_{t}$, which denotes the value of $J$ at time $t$. 
The first order condition for consumption is given by

$$
C_{t}=J_{W}^{-\psi}[(1-\gamma) J]^{\frac{1-\gamma \psi}{1-\gamma}} \beta^{\psi},
$$

which reduces to $C_{t}=\left(J / J_{W}\right)(1-\gamma) \beta$ when $\psi=1$.

The first order condition for portfolio choice is given by

$$
\alpha_{t}=\frac{-J_{W}}{W_{t} J_{W W}}\left(\frac{\mu_{t}-r}{\sigma_{S}^{2}}\right)-\frac{J_{W \mu}}{W_{t} J_{W W}}\left(\frac{\rho \sigma_{\mu}}{\sigma_{S}}\right) .
$$

Substitution of the first order conditions (25) and (26) into the Bellman equation (24) results in the following partial differential equation for the value function $J$ :

$$
\begin{aligned}
0= & f\left(J_{W}^{-\psi}\{(1-\gamma) J\}^{\frac{1-\gamma \psi}{1-\gamma}} \beta^{\psi}, J_{t}\right)-J_{W}\left\{J_{W}^{-\psi}[(1-\gamma) J]^{\frac{1-\gamma \psi}{1-\gamma}} \beta^{\psi}\right\} \\
& +J_{W} W_{t} r+J_{\mu} \kappa\left(\theta-\mu_{t}\right)+\frac{1}{2} J_{\mu \mu} \sigma_{\mu}^{2} \\
& -\frac{1}{2}\left\{\frac{J_{W}^{2}}{J_{W W}} \frac{\left(\mu_{t}-r\right)^{2}}{\sigma_{S}^{2}}+2 \frac{J_{W} J_{W \mu}}{J_{W W}} \frac{\rho \sigma_{\mu}\left(\mu_{t}-r\right)}{\sigma_{S}}+\frac{J_{W \mu}^{2}}{J_{W W}} \rho^{2} \sigma_{\mu}^{2}\right\} .
\end{aligned}
$$

Of course, the form of this equation depends on whether we consider the case $\psi \neq 1$ and use the normalized aggregator in (22), or we consider the case $\psi=1$ and use the normalized aggregator (23).Appendix B shows the partial differential equation that obtains in each case.

Campbell and Viceira (1999) claim that their discrete-time solution is exact for the case $\psi=1$ up to a discrete-time approximation to the log return on wealth, and note that this approximation becomes exact in continuous-time. The continuous-time model in this paper confirms their claim. We show in Appendix B that (27) has an exact analytical solution in the case $\psi=1$. This solution is

$$
J\left(W_{t}, \mu_{t}\right)=I\left(\mu_{t}\right) \frac{W_{t}^{1-\gamma}}{1-\gamma},
$$

with

$$
I\left(\mu_{t}\right)=\exp \left\{A_{0}+B_{0} \mu_{t}+\frac{C_{0}}{2} \mu_{t}^{2}\right\},
$$


where $A, B$, and $C$ are functions of the primitive parameters of the model describing investment opportunities and preferences.

In the more general case $\psi \neq 1$, there is no exact analytical solution to (27). However, we can still find an approximate analytical solution following the methods described in Campbell and Viceira (2002) and Chacko and Viceira (1999). We start by guessing that the value function in this case also has the form given in (28), with

$$
I\left(\mu_{t}\right)=H\left(\mu_{t}\right)^{-\left(\frac{1-\gamma}{1-\psi}\right)}
$$

Substitution of (30) into the Bellman equation (27) results in an ordinary differential equation for $H\left(\mu_{t}\right)$. This equation does not have an exact analytical solution in general. However, we show in Appendix B that taking a loglinear approximation to one of the terms in the equation results in a new equation for $H\left(\mu_{t}\right)$ that admits an analytical solution. The form of this solution is an exponential-quadratic function similar to (29):

$$
H\left(\mu_{t}\right)=\exp \left\{A_{1}+B_{1} \mu_{t}+\frac{C_{1}}{2} \mu_{t}^{2}\right\} .
$$

The term that we need to approximate in the ordinary differential equation for $H\left(\mu_{t}\right)$ is $\beta^{\psi} H\left(\mu_{t}\right)^{-1}$. Simple substitution of (28) and (31) into the first order condition (25) shows that this term is simply the optimal consumption-wealth ratio $C_{t} / W_{t}$. Thus this loglinearization is equivalent to loglinearizing the optimal consumption-wealth ratio around one particular point of the state space. Campbell and Viceira (2002) and Chacko and Viceira (1999) suggest approximating this term around the unconditional mean of the log consumption wealth-ratio. This choice has the advantage that the solution will be accurate if the log consumption-wealth is not too variable around its mean. Appendix B provides full details of this solution procedure. 


\subsection{Optimal portfolio choice}

The optimal portfolio policy of the investor obtains from substitution of the the solution for the value function into the first order condition (26). In the case $\psi=1$, substitution of (28)-(29) into the first order condition (26) gives

$$
\alpha_{t}=\left(\frac{1}{\gamma}\right) \frac{\mu_{t}-r}{\sigma_{S}^{2}}+\left(1-\frac{1}{\gamma}\right) \frac{\sigma_{\mu}}{\sigma_{S}} \rho\left(\mathcal{B}_{0}+\mathcal{C}_{0} \mu_{t}\right),
$$

where $\mathcal{B}_{0}=-B_{0} /(1-\gamma)$ and $\mathcal{C}_{0}=-C_{0} /(1-\gamma)$.

In the case $\psi \neq 1$, substitution of the approximate solution (28)-(30)-(31) into the first order condition (26) gives

$$
\alpha_{t}=\left(\frac{1}{\gamma}\right) \frac{\mu_{t}-r}{\sigma_{S}^{2}}+\left(1-\frac{1}{\gamma}\right) \frac{\sigma_{\mu}}{\sigma_{S}} \rho\left(\mathcal{B}_{1}+\mathcal{C}_{1} \mu_{t}\right),
$$

where $\mathcal{B}_{1}=-B_{1} /(1-\psi)$ and $\mathcal{C}_{1}=-C_{1} /(1-\psi)$. Appendix B shows that $\mathcal{B}_{1}$ and $\mathcal{C}_{1}$ do not depend on $\psi$, except through a loglinearization parameter.

Equations (32) and (33) show that the optimal allocation to stocks is a weighted average (with weights $1 / \gamma$ and $1-1 / \gamma$ ) of two terms, both of them linear in the expected return on stocks $\mu_{t}$. The first term is the myopic portfolio allocation to stocks, and the second term is the intertemporal hedging demand for stocks. The myopic portfolio allocation is proportional to $(1 / \gamma)$, so that it approaches zero as we consider increasingly risk averse investors. The intertemporal hedging component is proportional to $(1-1 / \gamma)$, so that one might be tempted to conclude that it does not approach zero in the limit as $\gamma \rightarrow 0$. However, we need to consider that $\mathcal{B}_{0}\left(\right.$ or $\left.\mathcal{B}_{1}\right)$ and $\mathcal{C}_{0}\left(\right.$ or $\mathcal{C}_{1}$ ) are also functions of $\gamma$. We can show that the limit of the overall expression approaches zero as $\gamma \rightarrow 0$. 


\subsection{Numerical calibration}

Section 3.3 derives the optimal portfolio rule for the continuous-time version of the discretetime model in Campbell and Viceira (1999). This portfolio rule is similar to the discrete time portfolio rule in their model ${ }^{3}$. We can use the parameter values given in Table I to calibrate the continuous-time portfolio rule (33), and compare the resulting mean allocations to those reported in Table III of Campbell and Viceira (2000). ${ }^{4}$ Note that the linearity of the optimal portfolio rule (32)-(33) implies that

$$
\mathrm{E}\left[\alpha_{t}\right]=\left(\frac{1}{\gamma}\right) \frac{\theta-r}{\sigma_{S}^{2}}+\left(1-\frac{1}{\gamma}\right) \frac{\sigma_{\mu}}{\sigma_{S}} \rho\left(\mathcal{B}_{i}+\mathcal{C}_{i} \theta\right), \quad i=0,1,
$$

where the first element of the sum is the mean myopic portfolio allocation, and the second element is the mean intertemporal hedging portfolio allocation.

Table II has a structure identical to Table III in Campbell and Viceira (2000) to facilitate comparison. Panel A in Table II shows mean optimal portfolio allocations implied by the parameter values given in Table I. These allocations are similar, but not identical, to those given in panel A of Table III in Campbell and Viceira (2000). This is a direct result of the nonlinearity in the time-aggregation of the variances and covariances of innovations $\left(\sigma_{S}, \sigma_{\mu}\right.$, $\rho)$, and the persistence parameter $\kappa$.

Panel B in Table II shows the percentage that the mean intertemporal hedging portfolio allocation

$$
\left(1-\frac{1}{\gamma}\right) \frac{\sigma_{\mu}}{\sigma_{S}} \rho\left(\mathcal{B}_{i}+\mathcal{C}_{i} \theta\right)
$$

represents over the total mean allocation (34). The numbers in this panel are very similar to those reported in Table III of Campbell and Viceira (2000), and support one of the main

\footnotetext{
${ }^{3}$ To see this, compare equation (33) with the expressions in Proposition 1 of Campbell and Viceira (1999).

${ }^{4}$ Appendix B shows that $\mathcal{B}_{1}$ and $\mathcal{C}_{1}$ depend on the loglinearization parameter $h_{1}=\mathrm{E}\left[c_{t}-w_{t}\right]$, which is endogenous. However, one can solve for $h_{1}$ using the simple numerical recursive algorithm described in Campbell and Viceira (1999).
} 
conclusions in Campbell and Viceira (1999): That given the historical experience in the US stock market, intertemporal hedging motives greatly increase the average demand for stocks by investors who are more risk averse than a logarithmic investor. For highly conservative investors, hedging may represent $90 \%$ or even more of the total mean demand for stocks.

\section{Conclusion}

This paper presents a continuous-time version of the model of optimal intertemporal portfolio choice and consumption with time-varying equity premium of Campbell and Viceira (1999). It shows that this model has an exact analytical solution when the investor has unit elasticity of intertemporal substitution in consumption and an approximate analytical solution otherwise. For calibration purposes, we also derive the discrete-time representation of the continuous-time VAR describing the asset return dynamics. This aggregation result is useful to recover the parameters of the model from discrete-time estimates. Our equivalence result shows that intuitive discrete-time representations of univariate continuous-time processes do not translate immediately to multivariate processes which are cross-sectionally correlated.

Our calibration results show that our portfolio choice model exhibits the same properties as its discrete-time counterpart. In particular, given the historical experience in the US stock market, intertemporal hedging motives greatly increase the average demand for stocks by investors who are more risk averse than a logarithmic investor. For highly conservative investors, hedging may represent $90 \%$ or even more of the total mean demand for stocks. 


\section{References}

Bergstrom, A.R., 1984, "Continuous Time Stochastic Models and Issues of Aggregation over Time", in Z. Griliches and M.D. Intriligator eds. Handbook of Econometrics Vol. 2, North-Holland, Amsterdam.

Campbell, John Y. and Albert S. Kyle, 1993, "Smart Money, Noise Trading, and Stock Price Behavior", Review of Economic Studies 60, 1-34.

Campbell, John Y. and Luis M. Viceira, 1999, "Consumption and Portfolio Decisions when Expected Returns are Time Varying", Quarterly Journal of Economics 114, 433-495.

Campbell, John Y. and Luis M. Viceira, 2000, "Consumption and Portfolio Decisions when Expected Returns are Time Varying: Erratum", unpublished paper, Harvard University.

Campbell, John Y. and Luis M. Viceira, 2002, Strategic Asset Allocation: Portfolio Choice for Long-Term Investors, Oxford University Press, New York, NY.

Chacko, George and Luis M. Viceira, 1999, "Dynamic Consumption and Portfolio Choice with Stochastic Volatility in Incomplete Markets," NBER Working Paper No. 7377.

Epstein, Lawrence and Stanley Zin, 1989, "Substitution, Risk Aversion, and the Temporal Behavior of Consumption and Asset Returns: A Theoretical Framework", Econometrica 57, 937-69.

Epstein, Lawrence and Stanley Zin, 1991, Substitution, Risk Aversion, and the Temporal Behavior of Consumption and Asset Returns: An Empirical Investigation", Journal of Political Economy 99, 263-286. 


\section{Appendix A}

We find $\exp (A s)$ by use of an induction proof. We first prove by induction that the matrix $A^{n}$ is given by

$$
A^{n}=\left(\begin{array}{cc}
0 & (-\kappa)^{n-1} \\
0 & (-\kappa)^{n}
\end{array}\right)
$$

To prove this result, assume that $A^{n}$ is given by (35). Then $A^{n+1}$ is given by

$$
\begin{aligned}
A^{n+1} & =A^{n} A \\
& =\left(\begin{array}{cc}
0 & (-\kappa)^{n-1} \\
0 & (-\kappa)^{n}
\end{array}\right)\left(\begin{array}{cc}
0 & 1 \\
0 & -\kappa
\end{array}\right) \\
& =\left(\begin{array}{cc}
0 & (-\kappa)^{n} \\
0 & (-\kappa)^{n+1}
\end{array}\right),
\end{aligned}
$$

which is the desired result.

The matrix $\exp (A s)$ is given by $I+A s+\cdots+A^{n} s^{n} / n !+\cdots$. Equation (35) allows us to write the exponential matrix as

$$
\begin{aligned}
\exp (A s) & =\left(\begin{array}{cc}
1 & 0 \\
0 & 1
\end{array}\right)+\frac{1}{n !} \sum_{n=1}^{\infty}\left(\begin{array}{cc}
0 & (-\kappa)^{n-1} s^{n} \\
0 & (-\kappa)^{n} s^{n}
\end{array}\right) \\
& =\left(\begin{array}{cc}
1 & \frac{1}{n !} \sum_{n=1}^{\infty}(-\kappa)^{n-1} s^{n} \\
0 & \frac{1}{n !} \sum_{n=0}^{\infty}(-\kappa s)^{n}
\end{array}\right) \\
& =\left(\begin{array}{cc}
1 & \frac{1}{n !} \sum_{n=1}^{\infty}(-\kappa)^{n-1} s^{n} \\
0 & \exp (-\kappa s)
\end{array}\right) .
\end{aligned}
$$

Now, notice that

$$
\frac{d}{d s}\left(\frac{1}{n !} \sum_{n=1}^{\infty}(-\kappa)^{n-1} s^{n}\right)=\frac{1}{n !} \sum_{n=0}^{\infty}(-\kappa s)^{n}=\exp (-\kappa s)
$$


so that

$$
\begin{aligned}
\frac{1}{n !} \sum_{n=1}^{\infty}(-\kappa)^{n-1} s^{n} & =\int \exp (-\kappa s) d s \\
& =\frac{-1}{\kappa} \exp (-\kappa s)+C
\end{aligned}
$$

Since at $s=0$ we have that $\left(\sum_{n=1}^{\infty}(-\kappa)^{n-1} s^{n}\right) / n !=0$, it follows that for the equation to hold at $s=0$ we must have that $C=\frac{1}{\kappa}$.

Therefore

$$
\frac{1}{n !} \sum_{n=1}^{\infty}(-\kappa)^{n-1} s^{n}=\frac{1}{\kappa}(1-\exp (-\kappa s)),
$$

from which it follows that we can write the matrix $\exp (A s)$ as

$$
\exp (A s)=\left(\begin{array}{cc}
1 & \frac{1}{\kappa}\left(1-e^{-\kappa s}\right) \\
0 & e^{-\kappa s}
\end{array}\right) .
$$

\section{Appendix B}

\subsection{Exact analytical solution when $\psi=1$}

Substitution of (28) and (29) into the Bellman equation (27) leads, after some simplification, to the following equation:

$$
\begin{aligned}
0= & -\frac{1}{1-\gamma} \beta\left\{A_{0}+B_{0} \mu_{t}+\frac{C_{0}}{2} \mu_{t}^{2}\right\}+\beta \log \beta+r-\beta \\
& +\frac{\kappa\left(\theta-\mu_{t}\right)}{1-\gamma}\left(B_{0}+C_{0} \mu_{t}\right)+\frac{\sigma_{\mu}^{2}}{2(1-\gamma)}\left\{C_{0}+\left(B_{0}+C_{0} \mu_{t}\right)^{2}\right\} \\
& +\frac{1}{2 \gamma}\left\{\frac{\left(\mu_{t}-r\right)^{2}}{\sigma_{S}^{2}}+2 \frac{\rho \sigma_{\mu}\left(\mu_{t}-r\right)}{\sigma_{S}}\left(B_{0}+C_{0} \mu_{t}\right)+\rho^{2} \sigma_{\mu}^{2}\left(B_{0}+C_{0} \mu_{t}\right)^{2}\right\} .
\end{aligned}
$$


We can now obtain $A_{0}, B_{0}$ and $C_{0}$ from the system of recursive equations that results from collecting terms in $\mu_{t}^{2}, \mu_{t}$, and constant terms:

$$
\begin{aligned}
0= & \frac{\sigma_{\mu}^{2}}{2}\left(1+\frac{1-\gamma}{\gamma} \rho^{2}\right) C_{0}^{2}+\left(-\frac{\beta}{2}-\kappa+\frac{1-\gamma}{\gamma} \frac{\rho \sigma_{\mu}}{\sigma_{S}}\right) C_{0}+\frac{1}{2 \gamma \sigma_{S}^{2}}, \\
0= & \kappa \theta C_{0}-\frac{1-\gamma}{\gamma} \frac{r}{\sigma_{S}^{2}}-\frac{1-\gamma}{\gamma} \frac{\rho r \sigma_{\mu}}{\sigma_{S}} C_{0} \\
& +\left(-\kappa-\beta+\sigma_{\mu}^{2}\left(1+\frac{1-\gamma}{\gamma} \rho^{2}\right) C_{0}+\frac{1-\gamma}{\gamma} \frac{\rho \sigma_{\mu}}{\sigma_{S}}\right) B_{0} \\
0= & -\beta A_{0}+(1-\gamma) \beta \log \beta+(1-\gamma)(r-\beta)+\frac{r^{2}}{2 \gamma \sigma_{S}^{2}}+\frac{\sigma_{\mu}^{2}}{2} C_{0} \\
& +\frac{\sigma_{\mu}^{2}}{2}\left(1+\frac{1-\gamma}{\gamma} \rho^{2}\right) B_{0}^{2}+\left(-\frac{1-\gamma}{\gamma} \frac{\sigma_{\mu}}{\sigma_{S}} \rho r+\kappa \theta\right) B .
\end{aligned}
$$

We can solve this system by solving equation (38) and then using the result to solve (39) and finally solve (40). Equation (38) is a quadratic equation whose only unknown is $C_{0}$. Thus it has two roots. Campbell and Viceira (1999) show that only one of them maximizes expected utility. This root is the one associated with the negative root of the discriminant of the equation. This is also the only root that ensures that $C_{0}=0$ when $\gamma=1$, that is, in the log utility case. This is a necessary condition for intertemporal hedging demand to be zero, as we know it must in the log utility case.

We can use these results to obtain the optimal portfolio policy of the investor from the first order condition (26), and the optimal consumption policy from the first order condition (25). The optimal portfolio policy is given in equatio (32) in text. It is easy to see that the optimal consumption policy is $C_{t} / W_{t}=\beta$, a constant consumption-wealth ratio equal to the rate of time preference. 


\subsection{Approximate analytical solution when $\psi \neq 1$}

Substitution of (28) and (30) into the Bellman equation (27) gives, after some simplification, the following ordinary differential equation:

$$
\begin{aligned}
0= & -\beta^{\psi} H^{-1}+\beta \psi+r(1-\psi)-\frac{H_{\mu}}{H} \kappa\left(\theta-\mu_{t}\right) \\
& +\frac{\sigma_{\mu}^{2}}{2}\left(-\frac{H_{\mu \mu}}{H}+\left(1+\frac{1-\gamma}{1-\psi}\right)\left(\frac{H_{\mu}}{H}\right)^{2}\right) \\
& +\frac{1-\psi}{2 \gamma}\left(\frac{\mu_{t}-r}{\sigma_{S}}\right)^{2}-\frac{1-\gamma}{\gamma} \frac{H_{\mu}}{H} \rho \sigma_{\mu}\left(\frac{\mu_{t}-r}{\sigma_{S}}\right)+\frac{1}{2} \frac{(1-\gamma)^{2}}{\gamma(1-\psi)}\left(\frac{H_{\mu \mu}}{H}\right)^{2} \rho^{2} \sigma_{\mu}^{2} .
\end{aligned}
$$

This ordinary differential equation does not have an exact analytical solution, unless $\psi=1$.

Though there does not exist an exact analytical solution to (41), we can still find an approximate analytical solution following the methods described in Campbell and Viceira (2002).and Chacko and Viceira (1999). First, we note that substitution of the solution guess (28)-(30) into the first order condition (25) gives

$$
\frac{C_{t}}{W_{t}}=\beta^{\psi} H\left(\mu_{t}\right)^{-1} .
$$

We can now use the following approximation for $\beta^{\psi} H^{-1}$ around the unconditional mean of the log consumption-wealth ratio:

$$
\begin{aligned}
\beta^{\psi} H\left(\mu_{t}\right)^{-1} & =\exp \left\{c_{t}-w_{t}\right\} \\
& \approx h_{0}+h_{1}\left(c_{t}-w_{t}\right) \\
& =h_{0}+h_{1}\left(\psi \log \beta-h_{t}\right),
\end{aligned}
$$

where $c_{t}=\log C_{t}, w_{t}=\log W_{t}, h_{t}=\log H\left(\mu_{t}\right)$, and

$$
\begin{aligned}
h_{1} & =\exp \left\{\mathrm{E}\left[c_{t}-w_{t}\right]\right\}, \\
h_{0} & =h_{1}\left(1-\log h_{1}\right) .
\end{aligned}
$$


Substitution of the approximation (42) for the first term of (41) transforms this ordinary differential equation into another one that has an exact solution, with the following exponential-quadratric form:

$$
H\left(\mu_{t}\right)=\exp \left\{A_{1}+B_{1} \mu_{t}+\frac{C_{1}}{2} \mu_{t}^{2}\right\} .
$$

The coefficients $A_{1}, B_{1}$, and $C_{1}$, can be obtained by solving the approximated Bellman equation

$$
\begin{aligned}
0= & -h_{0}-h_{1}\left\{\psi \log \beta-\left(A_{1}+B_{1} \mu_{t}+\frac{C_{1}}{2} \mu_{t}^{2}\right)\right\}+\beta \psi+r(1-\psi)-\kappa\left(\theta-\mu_{t}\right)\left(B_{1}+C_{1} \mu_{t}\right) \\
& +\frac{\sigma_{\mu}^{2}}{2}\left[-\left(\left(B_{1}+C_{1} \mu_{t}\right)^{2}+C_{1}\right)+\left(\frac{1-\gamma}{1-\psi}+1\right)\left(B_{1}+C_{1} \mu_{t}\right)^{2}\right]+\frac{1-\psi}{2 \gamma}\left(\frac{\mu_{t}-r}{\sigma_{S}}\right)^{2} \\
& -\frac{1-\gamma}{\gamma}\left(B_{1}+C_{1} \mu_{t}\right) \rho \sigma_{\mu}\left(\frac{\mu_{t}-r}{\sigma_{S}}\right)+\frac{1-\gamma}{2 \gamma}\left(\frac{1-\gamma}{1-\psi}\right)\left(B_{1}+C_{1} \mu_{t}\right)^{2} \rho^{2} \sigma_{\mu}^{2},
\end{aligned}
$$

which implies the following system of recursive equations:

$$
\begin{aligned}
0= & \frac{\sigma_{\mu}^{2}}{2} \frac{1-\gamma}{1-\psi}\left(1+\frac{1-\gamma}{\gamma} \rho^{2}\right) C_{1}^{2}+\left(\frac{h_{1}}{2}+\kappa-\frac{1-\gamma}{\gamma} \frac{\rho \sigma_{\mu}}{\sigma_{S}}\right) C_{1}+\frac{1-\psi}{2 \gamma \sigma_{S}^{2}} \\
0= & \kappa \theta C_{1}+\frac{1-\psi}{\gamma} \frac{r}{\sigma_{S}^{2}}-\frac{1-\gamma}{\gamma} \frac{\rho r \sigma_{\mu}}{\sigma_{S}} C_{1} \\
& +\left(\kappa+h_{1}+\sigma_{\mu}^{2} \frac{1-\gamma}{1-\psi}\left(1+\frac{1-\gamma}{\gamma} \rho^{2}\right) C_{1}-\frac{1-\gamma}{\gamma} \frac{\rho \sigma_{\mu}}{\sigma_{S}}\right) B_{1} \\
0= & h_{1} A_{1}-h_{0}-h_{1} \psi \log \beta+\beta \psi+r(1-\psi)+\frac{1-\psi}{2 \gamma} \frac{r^{2}}{\sigma_{S}^{2}}-\frac{\sigma_{\mu}^{2}}{2} C_{1} \\
& +\frac{\sigma_{\mu}^{2}}{2} \frac{1-\gamma}{1-\psi}\left(1+\frac{1-\gamma}{\gamma} \rho^{2}\right) B_{1}^{2}+\left(\frac{1-\gamma}{\gamma} \frac{\sigma_{\mu}}{\sigma_{S}} \rho r-\kappa \theta\right) B_{1} .
\end{aligned}
$$

We can solve this system by solving equation (45) and then using the result to solve (46) and finally solve (47). Equation (45) is a quadratic equation whose only unknown is $C$. Thus it has two roots. Campbell and Viceira (1999) show that only one of them maximizes expected utility. This root is the one associated with the positive root of the discriminant of 
the equation. Note also that this equation implies that $C /(1-\psi)$ does not depend on $\psi-$ except through the loglinearization parameter $h_{1}$ - which in turn implies, through equation (46), that $B /(1-\psi)$ does not depend on $\psi$ either. 


\section{TABLE I}

Continuous-Time Parameter Values

Implied by Campbell-Viceira (2000) VAR Estimates

Model:

$$
\begin{gathered}
d B_{t} / B_{t}=r d t \\
d S_{t} / S_{t}=\mu_{t} d t+\sigma_{S} d \tilde{Z}_{S}, \\
d \mu_{t}=\kappa\left(\theta-\mu_{t}\right) d t+\sigma_{\mu} d \tilde{Z}_{\mu}, \\
d \tilde{Z}_{S} d \tilde{Z}_{\mu}=\rho d t
\end{gathered}
$$

Parameter Values (at quarterly frequency):

$\begin{array}{cc}\boldsymbol{r} & 0.0818 \mathrm{e}-2 \\ \boldsymbol{\kappa} & 4.3875 \mathrm{e}-2 \\ \boldsymbol{\theta} & 1.3980 \mathrm{e}-2 \\ \boldsymbol{\sigma}_{S} & 7.8959 \mathrm{e}-2 \\ \boldsymbol{\sigma}_{\mu} & 0.5738 \mathrm{e}-2 \\ \boldsymbol{\rho} & -0.9626\end{array}$


TABLE II

Mean Optimal Percentage Allocation to Stocks and Percentage Mean Hedging Demand Over Mean Total Demand

\begin{tabular}{|c|c|c|c|c|c|c|c|c|}
\hline \multirow[t]{3}{*}{ R.R.A. } & \multicolumn{8}{|c|}{ E.I.S. } \\
\hline & \multicolumn{8}{|c|}{ (A) Mean optimal percentage allocation to stocks: } \\
\hline & $1 / .75$ & 1.00 & $1 / 1.5$ & $1 / 2$ & $1 / 4$ & $1 / 10$ & $1 / 20$ & $1 / 40$ \\
\hline 0.75 & 180.31 & 198.02 & 209.80 & 214.47 & 220.42 & 223.53 & 224.49 & 224.97 \\
\hline 1.00 & 211.12 & 211.12 & 211.12 & 211.12 & 211.12 & 211.12 & 211.12 & 211.12 \\
\hline 1.50 & 239.07 & 223.64 & 211.64 & 206.76 & 200.57 & 197.39 & 196.40 & 195.92 \\
\hline 2.00 & 248.85 & 227.85 & 210.53 & 203.36 & 194.22 & 189.52 & 188.07 & 187.36 \\
\hline 4.00 & 241.99 & 220.24 & 200.14 & 191.27 & 179.59 & 173.46 & 171.56 & 170.63 \\
\hline 10.0 & 183.24 & 173.55 & 163.80 & 159.06 & 152.29 & 148.46 & 147.23 & 146.62 \\
\hline 20.0 & 125.04 & 123.63 & 122.17 & 121.42 & 120.29 & 119.61 & 119.38 & 119.27 \\
\hline \multirow[t]{3}{*}{40.0} & 75.57 & 77.74 & 80.00 & 81.17 & 82.98 & 84.12 & 84.51 & 84.71 \\
\hline & \multicolumn{8}{|c|}{ (B) Fraction due to hedging demand (percentage): } \\
\hline & $1 / .75$ & 1.00 & $1 / 1.5$ & $1 / 2$ & $1 / 4$ & $1 / 10$ & $1 / 20$ & $1 / 40$ \\
\hline 0.75 & -56.12 & -42.16 & -34.17 & -31.26 & -27.71 & -25.94 & -25.39 & -25.13 \\
\hline 1.00 & 0.00 & 0.00 & 0.00 & 0.00 & 0.00 & 0.00 & 0.00 & 0.00 \\
\hline 1.50 & 41.13 & 37.06 & 33.49 & 31.93 & 29.82 & 28.69 & 28.34 & 28.16 \\
\hline 2.00 & 57.58 & 53.67 & 49.86 & 48.09 & 45.65 & 44.30 & 43.87 & 43.66 \\
\hline 4.00 & 78.19 & 76.03 & 73.63 & 72.40 & 70.61 & 69.57 & 69.23 & 69.07 \\
\hline 10.0 & 88.48 & 87.84 & 87.11 & 86.73 & 86.14 & 85.78 & 85.66 & 85.60 \\
\hline 20.0 & 91.56 & 91.46 & 91.36 & 91.31 & 91.22 & 91.17 & 91.16 & 91.15 \\
\hline 40.0 & 93.02 & 93.21 & 93.40 & 93.50 & 93.64 & 93.73 & 93.75 & 93.77 \\
\hline
\end{tabular}

